\title{
Erratum to: Emergent gauge bosons and dynamical symmetry breaking in a four-fermion Lifshitz model
}

\author{
T. Mariz ${ }^{1, \mathrm{a}}$, R. Moreira ${ }^{1, \mathrm{~b}}$, A. Yu. Petrov ${ }^{2, \mathrm{c}}$ \\ ${ }^{1}$ Instituto de Física, Universidade Federal de Alagoas, Maceió, Alagoas 57072-900, Brazil \\ 2 Departamento de Física, Universidade Federal da Paraíba, Caixa Postal 5008, João Pessoa, Paraíba 58051-970, Brazil
}

Received: 16 August 2019 / Accepted: 21 August 2019 / Published online: 30 August 2019

(C) The Author(s) 2019

Erratum to: Eur. Phys. J. C (2019) 79:550

https://doi.org/10.1140/epjc/s10052-019-7068-x

In this Erratum we correct some numerical coefficients for the low-energy effective action we obtained in our paper [1].

1. The Eq. (37) must be read as:

$$
\begin{aligned}
\mathcal{L}_{\text {eff }}= & \frac{g_{t}^{2}}{2} A_{0} A^{0}-\frac{\tilde{\alpha}_{2}}{2}\left(\partial_{0} A_{i} \partial^{0} A^{i}-\frac{6}{5} \partial_{0} A_{i} \partial^{i} A^{0}\right. \\
& \left.-\frac{6}{5} \partial_{i} A_{0} \partial^{0} A^{i}+\frac{36}{25} \partial_{i} A_{0} \partial^{i} A^{0}\right) \\
& -\frac{\tilde{\alpha}_{5} m^{4}}{2}\left(\partial_{i} A_{j} \partial^{i} A^{j}-\partial_{i} A_{j} \partial^{j} A^{i}\right) \\
& -\frac{9 \tilde{\alpha}_{2}}{50} \partial_{i} A_{0} \partial^{i} A^{0}-\frac{\tilde{\beta} m^{4} e^{2}}{4}\left(A_{i} A^{i}-\frac{a_{i} a^{i}}{e^{2}}\right)^{2},
\end{aligned}
$$

2. The Eqs. (41-44) must be read as:

$$
\begin{aligned}
& A_{0} \rightarrow \frac{5 m \tilde{\alpha}_{5}^{1 / 4}}{6 \tilde{\alpha}_{2}^{1 / 2}} A_{0}, \\
& A_{i} \rightarrow \frac{1}{m \tilde{\alpha}_{5}^{1 / 4}} A_{i}, \\
& \partial_{0} \rightarrow \frac{m^{2} \tilde{\alpha}_{5}^{1 / 4}}{\tilde{\alpha}_{2}^{1 / 2}} \partial_{0}, \\
& \partial_{i} \rightarrow \frac{1}{\tilde{\alpha}_{5}^{1 / 4}} \partial_{i},
\end{aligned}
$$

The original article can be found online at https://doi.org/10.1140/ epjc/s10052-019-7068-x.

\footnotetext{
a e-mail: tmariz@ fis.ufal.br

b e-mail: rmoreira@ fis.ufal.br

c e-mail: petrov@ fisica.ufpb.br
}

$$
\begin{aligned}
\mathcal{L}_{\mathrm{eff}}= & -\frac{m^{2}}{4} F_{\mu \nu} F^{\mu \nu}-\frac{m^{2}}{8}\left(\partial_{i} A_{0}\right)^{2}+\frac{g_{t}^{2}}{2} \frac{25 m^{2} \tilde{\alpha}_{5}^{1 / 2}}{36 \tilde{\alpha}_{2}} A_{0} A^{0} \\
& -\frac{\tilde{\beta} m^{2} e^{2}}{4 \tilde{\alpha}_{5}}\left(A_{i} A^{i}-\frac{a_{i} a^{i}}{e^{2}}\right)^{2} . \\
\mathcal{L}_{\mathrm{eff}}= & -\frac{m^{2}}{4} F_{\mu \nu} F^{\mu \nu}-\frac{\tilde{\beta} m^{2} e^{2}}{4 \tilde{\alpha}_{5}}\left(A_{i} A^{i}-\frac{a_{i} a^{i}}{e^{2}}\right)^{2}, \\
\mathcal{L}_{\mathrm{eff}}= & -\frac{m^{2}}{2} F_{0 i} F^{0 i}+\frac{m^{2}}{4} F_{i j} F^{i j} \\
& -\frac{\tilde{\beta}^{2} e^{2}}{4\left|\tilde{\alpha}_{5}\right|}\left(A_{i} A^{i}-\frac{a_{i} a^{i}}{e^{2}}\right)^{2} .
\end{aligned}
$$

None of physical conclusions of our paper are changed under these corrections.

Acknowledgements This work was partially supported by Conselho Nacional de Desenvolvimento Científico e Tecnológico (CNPq). The work by A. Yu. P. has been partially supported by the CNPq project 303783/2015-0.

Open Access This article is distributed under the terms of the Creative Commons Attribution 4.0 International License (http://creativecomm ons.org/licenses/by/4.0/), which permits unrestricted use, distribution, and reproduction in any medium, provided you give appropriate credit to the original author(s) and the source, provide a link to the Creative Commons license, and indicate if changes were made. Funded by SCOAP ${ }^{3}$.

\section{Reference}

1. T. Mariz, R. Moreira, A.Yu. Petrov, Eur. Phys. J. C 79, 550 (2019) 